\title{
Crystal structure of sodium trigallium pentaselenide, $\mathrm{NaGa}_{3} \mathrm{Se}_{5}$
}

\author{
L. Kienle and H. J. Deiseroth \\ Universität Siegen, Anorganische Chemie, D-57068 Siegen, Germany
}

Received February 13, 1997. transferred to 1st update of database ICSD in 1998, CSD-No. 402880

Source of material: Single crystals of the new nonmetallic filledup $\beta$-Mn phase $\mathrm{NaGa}_{3} \mathrm{Se}_{5}$ could be prepared by direct reaction of stoichiometric mixtures of the elements at $1030 \mathrm{~K}$.

Compared to all known chalcogenides with this structure type (for example $\mathrm{Ag}_{2} \mathrm{Ga}_{6} \mathrm{Te}_{10}, \mathrm{SnGa}_{6} \mathrm{Te}_{10}, \mathrm{Ga}_{7} \mathrm{Te}_{10}$ see refs. 1-3), the $\mathrm{Ga}^{3+}$ are ordered in a hitherto unknown way. The ordering is characterized by a framework of comer-, edge- and face-sharing

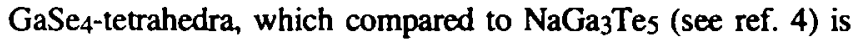
much more complex. This is indicated by the lower symmetry of this space group and by the increased unit cell volume.

Ga6 Na2Se10, orthorhombic, $P 22_{1} 2_{1}$ (No. 19), $a=9.730$ (2) $\AA$, $b=26.939(5) \AA, c=13.597(3) \AA, V=3564.0 \AA^{3}, Z=8, R(F)=0.036$, $R_{w}\left(F^{2}\right)=0.084$.

Table 1. Parameters used for the $\mathrm{X}$-ray data collection

Crystal:

Wavelength:

$\mu$ :

Diffractometer:

Scan mode:

Tmeasurement:

$2 \theta_{\max }:$

$\mathrm{N}(\boldsymbol{h k})_{\text {unique: }}$

Criterion for $l_{0}$ :

$N(\text { param })_{\text {refined: }}$

Programs:

\author{
red, fragment of a needle, \\ size $0.05 \times 0.06 \times 0.09 \mathrm{~mm}$ \\ Mo $K_{\alpha}$ radiation $(0.71069 \AA)$ \\ $293.90 \mathrm{~cm}^{-1}$ \\ Enraf-Nonius CAD4 \\ $\omega / 2 \theta$ \\ $293 \mathrm{~K}$ \\ $50^{\circ}$ \\ 4923 \\ $I_{0}>2 \sigma\left(I_{0}\right)$ \\ 361
} SHELXL-93, NRCVAX

Table 2. Final atomic coordinates and displacement parameters (in $\AA^{2}$ )

\begin{tabular}{|c|c|c|c|c|c|c|c|c|c|c|}
\hline Atom & Site & $x$ & $y$ & $z$ & $U_{11}$ & $U_{22}$ & $U_{33}$ & $U_{12}$ & $U_{13}$ & $U_{23}$ \\
\hline $\operatorname{Se}(1)$ & $4 a$ & $0.8167(2)$ & $0.43840(6)$ & $0.5610(1)$ & $0.020(1)$ & $0.012(1)$ & $0.0192(9)$ & $0.0016(8)$ & $-0.0001(7)$ & $-0.0002(6)$ \\
\hline $\operatorname{Se}(2)$ & $4 a$ & $0.4225(2)$ & $0.41967(6)$ & $0.4565(1)$ & $0.018(1)$ & $0.015(1)$ & $0.029(1)$ & $0.0010(8)$ & $0.0003(7)$ & $-0.0023(7)$ \\
\hline $\operatorname{Se}(3)$ & $4 a$ & $0.3205(2)$ & $0.51971(7)$ & $0.1492(1)$ & $0.020(1)$ & $0.020(1)$ & $0.0217(9)$ & $0.0022(9)$ & $-0.0001(7)$ & $-0.0005(7)$ \\
\hline $\operatorname{Se}(4)$ & $4 a$ & $0.4857(2)$ & $0.40138(7)$ & $0.7507(1)$ & $0.026(1)$ & $0.016(1)$ & $0.0196(9)$ & $0.0019(9)$ & $0.0028(7)$ & $0.0019(7)$ \\
\hline $\operatorname{Se}(5)$ & $4 a$ & $0.5541(2)$ & $0.42089(6)$ & $0.0262(1)$ & $0.025(1)$ & $0.018(1)$ & $0.0213(9)$ & $-0.0035(9)$ & $-0.0010(7)$ & $0.0008(7)$ \\
\hline $\operatorname{Se}(6)$ & $4 a$ & $0.6883(2)$ & $0.51556(7)$ & $0.3500(1)$ & $0.025(1)$ & $0.023(1)$ & $0.0233(9)$ & $-0.0012(9)$ & $-0.0020(7)$ & $0.0025(7)$ \\
\hline $\operatorname{Se}(7)$ & $4 a$ & $0.1665(2)$ & $0.43795(6)$ & $-0.0825(1)$ & $0.019(1)$ & $0.013(1)$ & $0.0182(9)$ & $-0.0006(8)$ & $0.0008(7)$ & $0.0001(7)$ \\
\hline $\operatorname{Se}(8)$ & $4 a$ & $-0.0657(2)$ & $0.33284(6)$ & $0.0345(1)$ & $0.022(1)$ & $0.016(1)$ & $0.0201(9)$ & $-0.0032(8)$ & $0.0017(7)$ & $-0.0021(7)$ \\
\hline $\operatorname{Se}(9)$ & $4 a$ & $-0.0112(2)$ & $0.34722(7)$ & $0.7521(1)$ & $0.022(1)$ & $0.016(1)$ & $0.0179(9)$ & $-0.0022(8)$ & $-0.0012(7)$ & $0.0011(7)$ \\
\hline $\operatorname{Se}(10)$ & $4 a$ & $0.1842(2)$ & $0.23372(6)$ & $0.1655(1)$ & $0.020(1)$ & $0.012(1)$ & $0.0206(9)$ & $-0.0019(8)$ & $-0.0029(7)$ & $0.0025(7)$ \\
\hline $\operatorname{Se}(11)$ & $4 a$ & $0.2473(2)$ & $0.37588(7)$ & $0.1877(1)$ & $0.023(1)$ & $0.016(1)$ & $0.023(1)$ & $0.0047(9)$ & $-0.0015(7)$ & $-0.0029(7)$ \\
\hline $\operatorname{Se}(12)$ & $4 a$ & $0.0802(2)$ & $0.46107(6)$ & $0.3633(1)$ & $0.019(1)$ & $0.016(1)$ & $0.0173(9)$ & $-0.0018(9)$ & $-0.0017(7)$ & $0.0000(6)$ \\
\hline $\operatorname{Se}(13)$ & $4 a$ & $-0.0695(2)$ & $0.46764(6)$ & $0.1302(1)$ & $0.020(1)$ & $0.015(1)$ & $0.0207(9)$ & $-0.0008(9)$ & $-0.0003(7)$ & $-0.0006(6)$ \\
\hline $\operatorname{Se}(14)$ & $4 a$ & $0.7487(2)$ & $0.37379(6)$ & $0.3004(1)$ & $0.025(1)$ & $0.014(1)$ & $0.0212(9)$ & $-0.0019(9)$ & $-0.0029(7)$ & $0.0018(7)$ \\
\hline $\operatorname{Se}(15)$ & $4 a$ & $-0.2111(2)$ & $0.23525(7)$ & $0.3468(1)$ & $0.019(1)$ & $0.014(1)$ & $0.024(1)$ & $0.0028(8)$ & $0.0012(7)$ & $0.0022(7)$ \\
\hline $\operatorname{Se}(16)$ & $4 a$ & $0.0674(2)$ & $0.33568(6)$ & $0.4575(1)$ & $0.020(1)$ & $0.015(1)$ & $0.0255(9)$ & $0.0002(8)$ & $-0.0014(7)$ & $0.0026(7)$ \\
\hline $\operatorname{Se}(17)$ & $4 a$ & $0.6722(2)$ & $0.31506(6)$ & $0.5751(1)$ & $0.018(1)$ & $0.016(1)$ & $0.0191(9)$ & $-0.0009(8)$ & $-0.0004(7)$ & $-0.0003(7)$ \\
\hline $\operatorname{Se}(18)$ & $4 a$ & $0.4322(2)$ & $0.28739(6)$ & $0.3810(1)$ & $0.018(1)$ & $0.013(1)$ & $0.0179(9)$ & $0.0001(8)$ & $-0.0002(7)$ & $-0.0016(6)$ \\
\hline $\operatorname{Se}(19)$ & $4 a$ & $0.3142(2)$ & $0.30803(6)$ & $-0.0747(1)$ & $0.017(1)$ & $0.014(1)$ & $0.0177(9)$ & $-0.0006(8)$ & $-0.0005(7)$ & $-0.0002(6)$ \\
\hline $\operatorname{Se}(20)$ & $4 a$ & $0.5645(2)$ & $0.28636(6)$ & $0.1089(1)$ & $0.017(1)$ & $0.011(1)$ & $0.0160(9)$ & $-0.0005(8)$ & $-0.0009(6)$ & $-0.0006(6)$ \\
\hline $\mathrm{Ga}(1)$ & $4 a$ & $0.6040(2)$ & $0.47402(7)$ & $0.4932(1)$ & $0.018(1)$ & $0.018(1)$ & $0.022(1)$ & $-0.0003(9)$ & $0.0008(7)$ & $-0.0009(8)$ \\
\hline $\mathrm{Ga}(2)$ & $4 a$ & $0.2558(2)$ & $0.48281(7)$ & $0.4827(1)$ & $0.020(1)$ & $0.016(1)$ & $0.019(1)$ & $0.0006(9)$ & $-0.0009(7)$ & $0.0003(7)$ \\
\hline $\mathrm{Ga}(3)$ & $4 a$ & $0.0972(2)$ & $0.51707(7)$ & $0.2210(1)$ & $0.021(1)$ & $0.017(1)$ & $0.017(1)$ & $-0.001(1)$ & $0.0012(7)$ & $-0.0015(7)$ \\
\hline $\mathrm{Ga}(4)$ & $4 a$ & $0.3857(2)$ & $0.39471(7)$ & $-0.0899(1)$ & $0.020(1)$ & $0.016(1)$ & $0.021(1)$ & $-0.0015(9)$ & $0.0006(8)$ & $0.0003(8)$ \\
\hline $\mathrm{Ga}(5)$ & $4 a$ & $0.6073(2)$ & $0.49699(7)$ & $-0.0571(1)$ & $0.019(1)$ & $0.019(1)$ & $0.022(1)$ & $-0.0016(9)$ & $-0.0005(8)$ & $-0.0001(8)$ \\
\hline $\mathrm{Ga}(6)$ & $4 a$ & $0.0796(2)$ & $0.40155(7)$ & 0.0699 (1) & $0.022(1)$ & $0.015(1)$ & $0.021(1)$ & $0.0000(9)$ & $0.0009(8)$ & $-0.0005(7)$ \\
\hline $\mathrm{Ga}(7)$ & $4 a$ & $0.5643(2)$ & $0.19522(7)$ & $0.1032(1)$ & $0.020(1)$ & $0.013(1)$ & $0.017(1)$ & $0.0012(9)$ & $0.0016(8)$ & $-0.0005(7)$ \\
\hline $\mathrm{Ga}(8)$ & $4 a$ & $0.3325(2)$ & $0.21550(7)$ & $0.3005(1)$ & $0.018(1)$ & $0.014(1)$ & $0.015(1)$ & $-0.0013(9)$ & $0.0000(7)$ & $0.0005(7)$ \\
\hline $\mathrm{Ga}(9)$ & $4 a$ & $0.3146(2)$ & $0.30195(7)$ & $0.1049(1)$ & $0.021(1)$ & $0.013(1)$ & $0.018(1)$ & $0.0019(9)$ & $-0.0004(8)$ & $0.0005(8)$ \\
\hline $\mathrm{Ga}(10)$ & $4 a$ & $0.6386(2)$ & $0.29614(7)$ & $0.2775(1)$ & $0.020(1)$ & $0.016(1)$ & $0.016(1)$ & $-0.0002(9)$ & $-0.0020(7)$ & $0.0001(8)$ \\
\hline $\mathrm{Ga}(11)$ & $4 a$ & $0.1139(2)$ & $0.25704(7)$ & $0.5282(1)$ & $0.021(1)$ & $0.016(1)$ & $0.018(1)$ & $0.0020(9)$ & $0.0012(8)$ & $-0.0003(8)$ \\
\hline $\mathrm{Ga}(12)$ & $4 a$ & $-0.1715(2)$ & $0.36026(7)$ & $0.4659(1)$ & $0.019(1)$ & $0.017(1)$ & $0.019(1)$ & $-0.002(1)$ & $-0.0001(8)$ & $0.0009(7)$ \\
\hline $\mathrm{Na}(1)$ & $4 a$ & $0.0023(7)$ & $0.3091(3)$ & $0.2516(5)$ & $0.024(5)$ & $0.040(6)$ & $0.023(4)$ & $0.006(4)$ & $0.006(3)$ & $0.002(3)$ \\
\hline $\mathrm{Na}(2)$ & $4 a$ & $0.2579(8)$ & $0.6286(3)$ & $0.1301(5)$ & $0.046(6)$ & $0.016(5)$ & $0.029(4)$ & $-0.005(4)$ & $0.005(3)$ & $0.004(3)$ \\
\hline $\mathrm{Na}(3)$ & $4 a$ & $0.5016(8)$ & $0.4400(3)$ & $0.2404(5)$ & $0.045(5)$ & $0.025(5)$ & $0.032(4)$ & $0.008(4)$ & $-0.002(4)$ & $-0.004(3)$ \\
\hline $\mathrm{Na}(4)$ & $4 a$ & $0.2535(7)$ & $0.1220(3)$ & $0.1175(5)$ & $0.029(5)$ & $0.022(5)$ & $0.038(5)$ & $-0.006(4)$ & $-0.001(3)$ & $-0.005(3)$ \\
\hline
\end{tabular}


Acknowledgment. This work was supported by the Deutsche Forschungsgemeinschaft.

\section{References}

1. Panzer, B.; Range, K. J.;Zabel, M.: Hochdrucksynthese und Kristallstruktur von $\mathrm{Ag}_{2} \mathrm{Ga}_{6} \mathrm{Te}_{10}$. J. Less-Common Met. 106 (1985) 305-314.

2. Deiseroth, H. J.; Müller, H. D.: Structural Relations in the Family of Nonmetallic Filled $\beta$-Manganese Phases: The new Members AGa6Te 10 (A: Sn, Pb) and Pbln6Te10. Z. anorg. allg. Chem. 622 (1996) 405-410.
3. Deiseroth, H. J.; Müller, H. D.: Crystal structures of heptagallium decatelluride Ga7Te 10 and heptaindium decatelluride $\operatorname{In}_{7} \mathrm{Te}_{10}$ - Z. Kristallogr. 210 (1995) 57-58.

4. Kienle, L.; Deiseroth, H. J.: Crystal structure of natrium trigallium pentatelluride NaGa3Te5. Z. Kristallogr. 211 (1996) 629

5. Sheldrick, G. M.: SHELXL-93. Program for refining crystal structures University of Göttingen, Germany 1993.

6. Gabe, E. J.; Lee, F. L.; Le Page, Y.: The NRCVAX crystal structure system Crystallograhic computing 3. Cleardon Press, Oxford 1985. 